\title{
Effects of Geometric Parameters on Jet Attachment of Counter-Flow Thrust Vectoring Nozzle
}

\author{
Xiang WANG ${ }^{1, a}$, Zhao-Miao LIU ${ }^{1}$, *, Hui-Long ZHENG ${ }^{1,2}$, Tan ZHANG ${ }^{2}$ \\ ${ }^{1}$ College of Mechanical Engineering and Applied Electronics Technology, Beijing University of \\ Technology, Beijing 100124, China \\ ${ }^{2}$ Aviation Key Laboratory of Science and Technology on Precision Manufacturing, Precision \\ Engineering Institute for Aircraft Industry, Beijing 100076, China
}

Keywords: Jet attachment, Slot height, Axial length, Counter-flow, Vectoring nozzle.

\begin{abstract}
Numerical simulation was applied to study the effects of changing geometric parameters, which were slot height $G$ and axial length of outer collar $L$, on jet attachment of counter-flow thrust vectoring nozzle in zero attack angle and subsonic conditions. Combined internal flow characteristics of different suction outlet pressure with pressure distribution of upper wall of outer collar, the range of suction outlet pressure when main flow is attached to upper wall is obtained. Moreover, the attachment point moves towards the inlet of outer collar with the increase of suction outlet pressure when attachment appears. And 0.2 bar was set as the work condition of suction outlet pressure for subsequent study. Critical values for $G$ (between $0.017 \mathrm{~m}$ and $0.02 \mathrm{~m}$ ) and $L$ (between $0.19 \mathrm{~m}$ and $0.2 \mathrm{~m}$ ) were confirmed. Attachment can be avoided and the clearance between main flow and upper wall grows larger when increasing $G$ or decreasing $L$, separately.
\end{abstract}

\section{Introduction}

As one of the key techniques of aviation technology, thrust vectoring control could meet the rising demand of aerial defence proposed by modern aviation weapon performance and space-aeronautics incorporation. Among them, the mechanical thrust vectoring control mostly adopts hydraulic or mechanical components and has been used widely [1, 2]. However, these complicated components and structures increase the complexity and cost of the system and weaken its reliability. By contrast, the fluid thrust vectoring control needs few moving parts and its mass and cost are reduced in turn. As one fluid control method, counter-flow thrust vectoring provides more advantages, low thrust loss and secondary mass flow rate, needless of secondary flow with high pressure and valid for both subsonic and supersonic conditions [3, 4].

Counter-flow thrust vectoring nozzle shows varied flow patterns and vectoring characteristics for different geometric parameters or working conditions. The numerical results obtained by Hunter are in good agreement with their experimental data and he also revealed the relationship between the counter-flow shearing layers and thrust vectoring [5]. Flamm experimentally studied the internal performance of the nozzle with the compression ratio in the range of 3.5-10 and the influence of nozzle structure and slot width on vectoring characteristics was revealed [6]. Wang numerically studied the internal flow patterns with unsteady phenomena. One unsteadiness was induced by boundary conditions as a result of flow hysteresis after jet attachment, the other self-induced unsteadiness was caused by the periodic oscillation of the main flow [7, 8]. Liu numerically researched vectoring characteristics with different aerodynamic parameters of Mach number and attack angle or geometric parameters of slot width, horizontal height and collar length $[9,10]$.

Most studies mentioned focused on internal flow patterns and vectoring characteristics under varied conditions and less attention was paid to the topic of jet attachment and its evasion method. But once attached, counter-flow thrust vectoring nozzle couldn't be controlled. Hence it is necessary to conduct in-depth study of the formation reason of jet attachment and its evasion method. This paper numerically researches the flow pattern of the nozzle when the main flow attached and verifies the effectiveness of the evasion method by varying the geometric parameters. 


\section{Model and Method}

\section{Calculation Model and Geometric Parameters}

The calculation model in this paper is based on the scaled model of the two-dimensional nozzle in reference [2] and modified according to the variables, as shown in Figure 1. The geometric parameters of the basic model are as follows. The main nozzle adopts Laval nozzle with cross-section height $\mathrm{H}$ $=0.04 \mathrm{~m}$, expansion ratio (which means the ratio of the area of outlet to the area of throat) $\pi=1.69$, inclination angle $14^{\circ}$. Oblique angle $\theta=30^{\circ}$, slot width $\mathrm{G}=0.425 \mathrm{H}=0.017 \mathrm{~m}$, suction angle $\varphi=14^{\circ}$, lateral width $\mathrm{C}=1.5 \mathrm{H}=0.06 \mathrm{~m}$, axial length $\mathrm{L}=5 \mathrm{H}=0.2 \mathrm{~m}$. The variables are $\mathrm{G}=(0.014,0.017,0.02$, $0.026) \mathrm{m}, \mathrm{L}=(0.17,0.18,0.19,0.2,0.23) \mathrm{m}$.

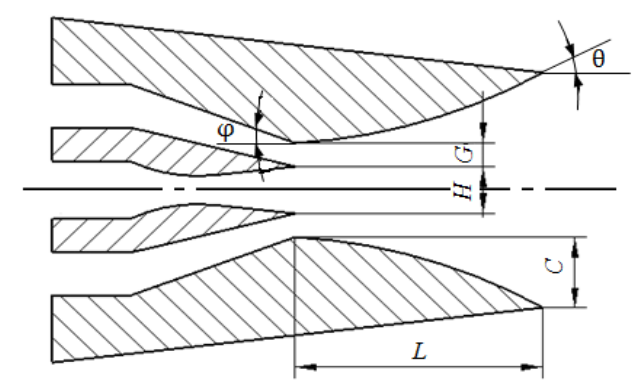

Figure 1. Model and geometric parameters.

\section{Mesh}

All flow fields of calculation are divided into five parts, main flow field, counter-flow secondary flow field, co-flow secondary flow field, collar vectoring field and far-field flow field, occupied by structured grid, as shown in Figure 2. Both the upside and downside of outflow far-field range are $30 \mathrm{H}$ while the right side range is $40 \mathrm{H}$. The counter-flow layer and co-flow shear layer are both partial grid refined and the near wall zone is also partial grid refined. The partial enlargement of mesh is shown in Figure 3.

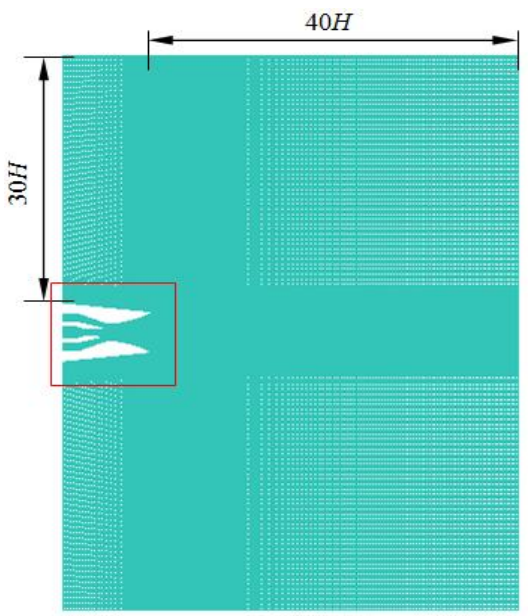

Figure 2. Calculation mesh.

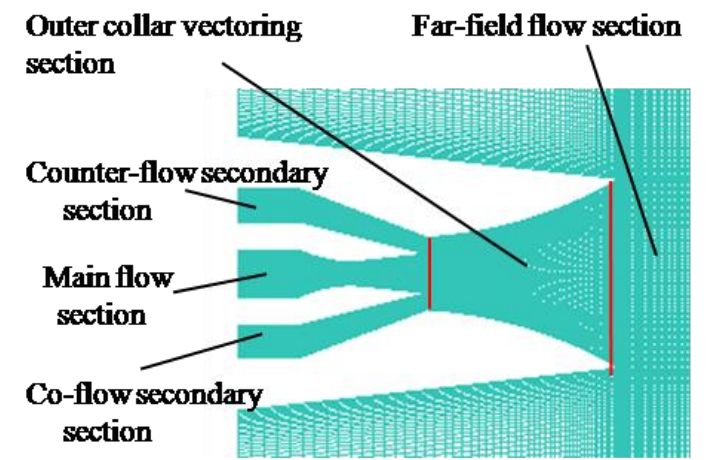

Figure 3. Partial enlargement of mesh.

\section{Boundary Condition}

The variation of temperature is neglected and all working temperature is set to be $300 \mathrm{~K}$. The total pressure of the main nozzle inlet is set to $8 \mathrm{~atm}$, the total pressure of both the co-flow secondary nozzle inlet and far-field boundary are set to be 1atm as they connect directly to external atmosphere. The total pressure of counter-flow secondary nozzle outlet changes as $(0.1,0.15,0.2,0.25,0.3,0.4,0.5$, 0.6)atm. The attack angel of far-field flow is $0^{\circ}$ and its Mach number is 0.7 . 


\section{Results and Discussion}

\section{Outlet Pressure of Counter-Flow}

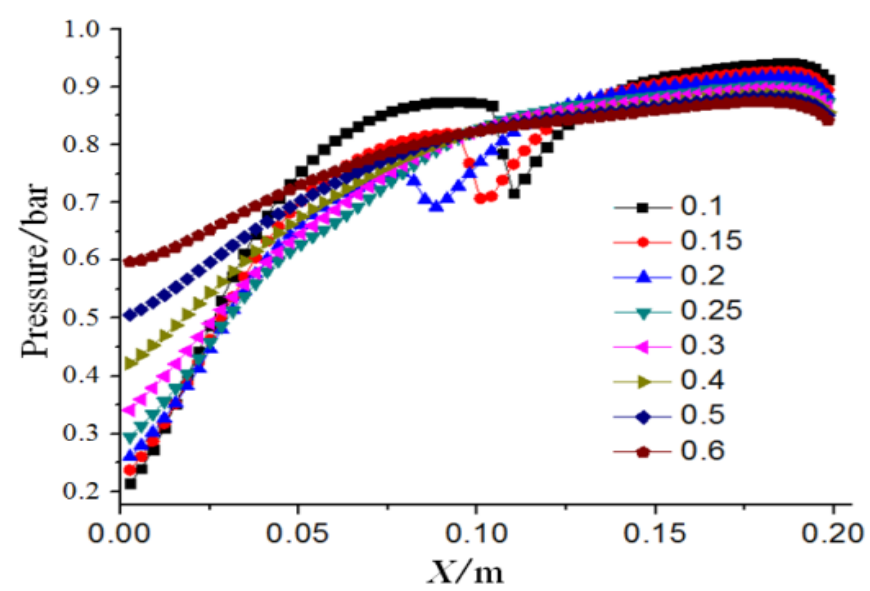

Figure 4. The pressure distribution of upper wall of outer collar with different outlet pressure of counter-flow

In this part, the geometric parameters of the basic model are kept the same and only the outlet pressure of counter-flow secondary nozzle is varied. The pressure distribution of upper wall of outer collar with different outlet pressure of counter-flow is shown in Figure 4. When the outlet pressure is 0.1bar, 0.15 bar or $0.2 \mathrm{bar}$, a sudden downward change is present in the pressure distribution profile. Moreover, the sudden change spot is around $0.1 \mathrm{~m}$ of outer collar and moves towards the inlet of outer collar as the suction pressure increases, which means that the corresponding $\mathrm{X}$ value decreases. Combined with the streamline diagram shown in Figure 5, it is indicated that the main flow is attached to outer collar at the sudden change spot. So the jet attachment point moves towards the inlet of outer collar as suction pressure increases.
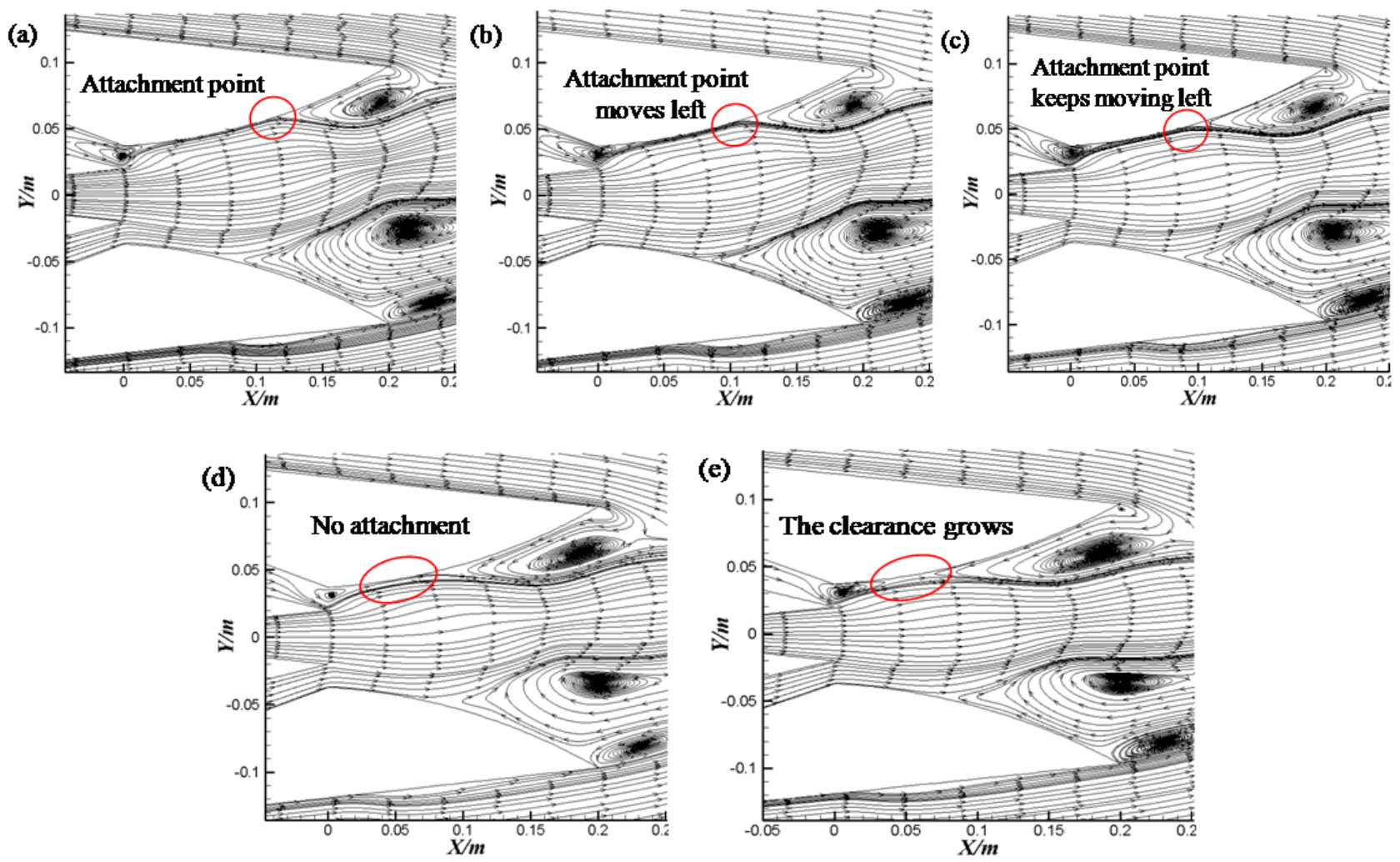

Figure 5. The streamline diagrams for different suction pressure with (a) $0.1 \mathrm{bar}$, (b) $0.15 \mathrm{bar}$, (c)

$0.2 \mathrm{bar},(\mathrm{d}) 0.25 \mathrm{bar},(\mathrm{e}) 0.4 \mathrm{bar}$. 
The main flow expands to both sides with the influence of pressure gradient when it flows into outer collar and the secondary flows are deflected along the collar walls, respectively. At low suction pressure (equal or less than 0.2bar), the pressure gradient at the upper collar wall is relatively large and the expansion range is wide. So the main flow tends to be attached. As suction pressure turns larger, the resistance of the main flow expansion grows larger and the main flow moves away from the upper collar wall laterally and no attachment is present any more. On the other hand, the influence scope of the entrained outflow is larger with larger suction pressure. And the attachment point moves towards the inlet of outer collar when the main flow is attached at suction pressure of $0.1 \mathrm{bar}, 0.15 \mathrm{bar}$, 0.2 bar.

So it is shown that a critical suction pressure is present for the jet attachment and it falls between 0.2 bar and 0.25 bar for the model in this paper.

\section{Slot Width G}

In order to investigate the influence of slot width $\mathrm{G}$ on jet attachment, the other geometric parameters are the same with the basic model and the suction pressure is kept to be $0.2 \mathrm{bar}$. It is shown in Figure 6(a) that the main flow is still attached when $\mathrm{G}$ decreases to $0.014 \mathrm{~m}$ while counter-flow appears at the upper collar wall when $\mathrm{G}$ increases to $0.02 \mathrm{~m}$ or $0.026 \mathrm{~m}$. And the clearance grows larger with a larger G. As the main flow expands to both sides, the space for deflection is not enough when $G$ is less than a critical value. With a larger slot width, the main flow expands completely without contacting with the upper wall and jet attachment is avoided. In addition, the main flow is pushed away from the wall by the entrained outflow and the expansion of the main flow is restrained. Outflow is entrained into the outer collar along the upper collar wall and secondary counter-flow is formed.

It is indicated that a critical slot width is present which falls between $0.017 \mathrm{~m}$ and $0.02 \mathrm{~m}$ for the model and jet attachment can be avoided by increasing the slot width.
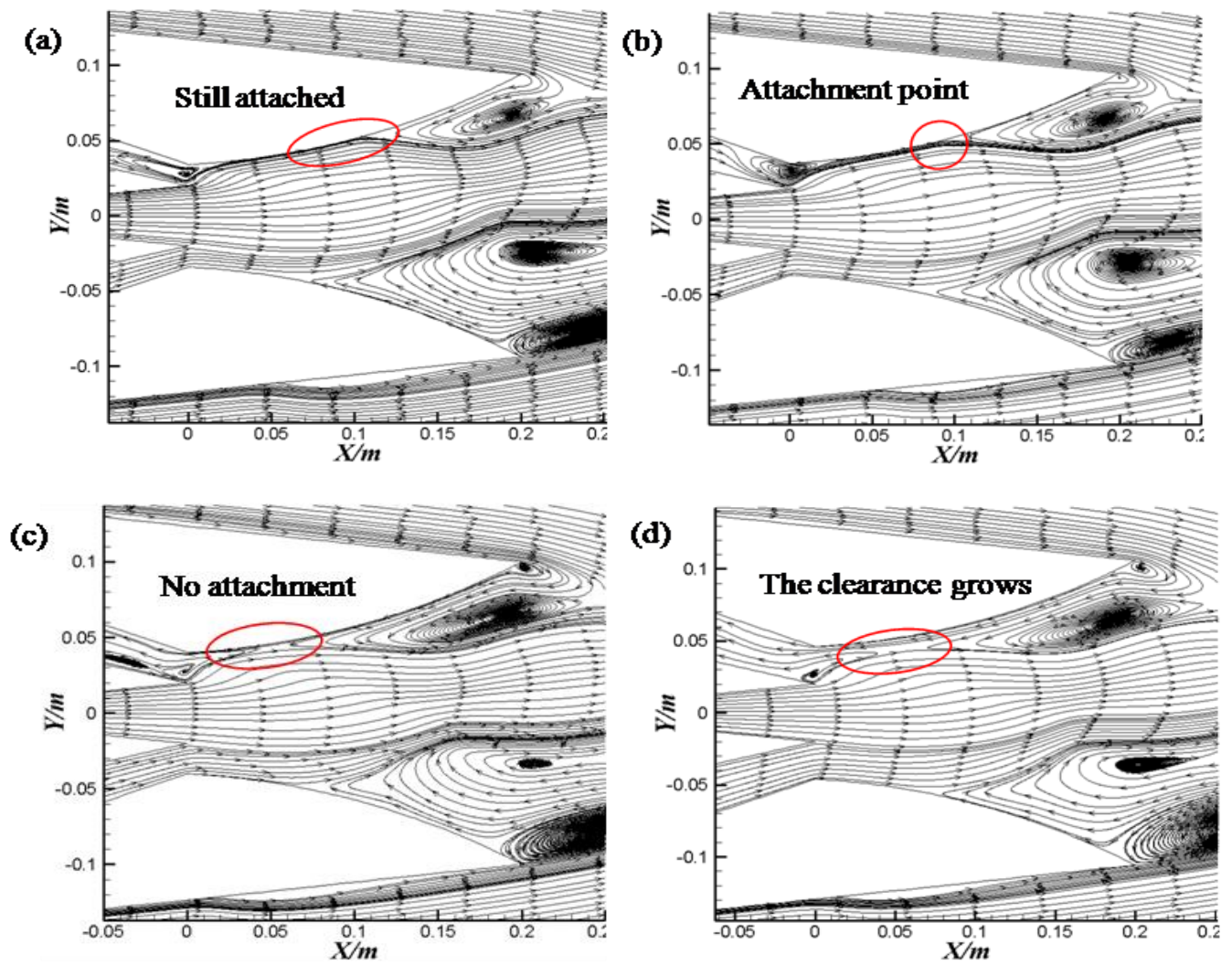

Figure 6. The streamline diagrams for different slot width $\mathrm{G}$ with (a) $0.014 \mathrm{~m}$, (b) $0.017 \mathrm{~m}$, (c) $0.02 \mathrm{~m},(\mathrm{~d}) 0.026 \mathrm{~m}$. 


\section{Axial Length of Outer Collar L}

To investigate the influence of axial length of outer collar $\mathrm{L}$ on jet attachment, the other geometric parameters are the same with the basic model and the suction pressure is also kept to be $0.2 \mathrm{bar}$ and only $\mathrm{L}$ is varied.
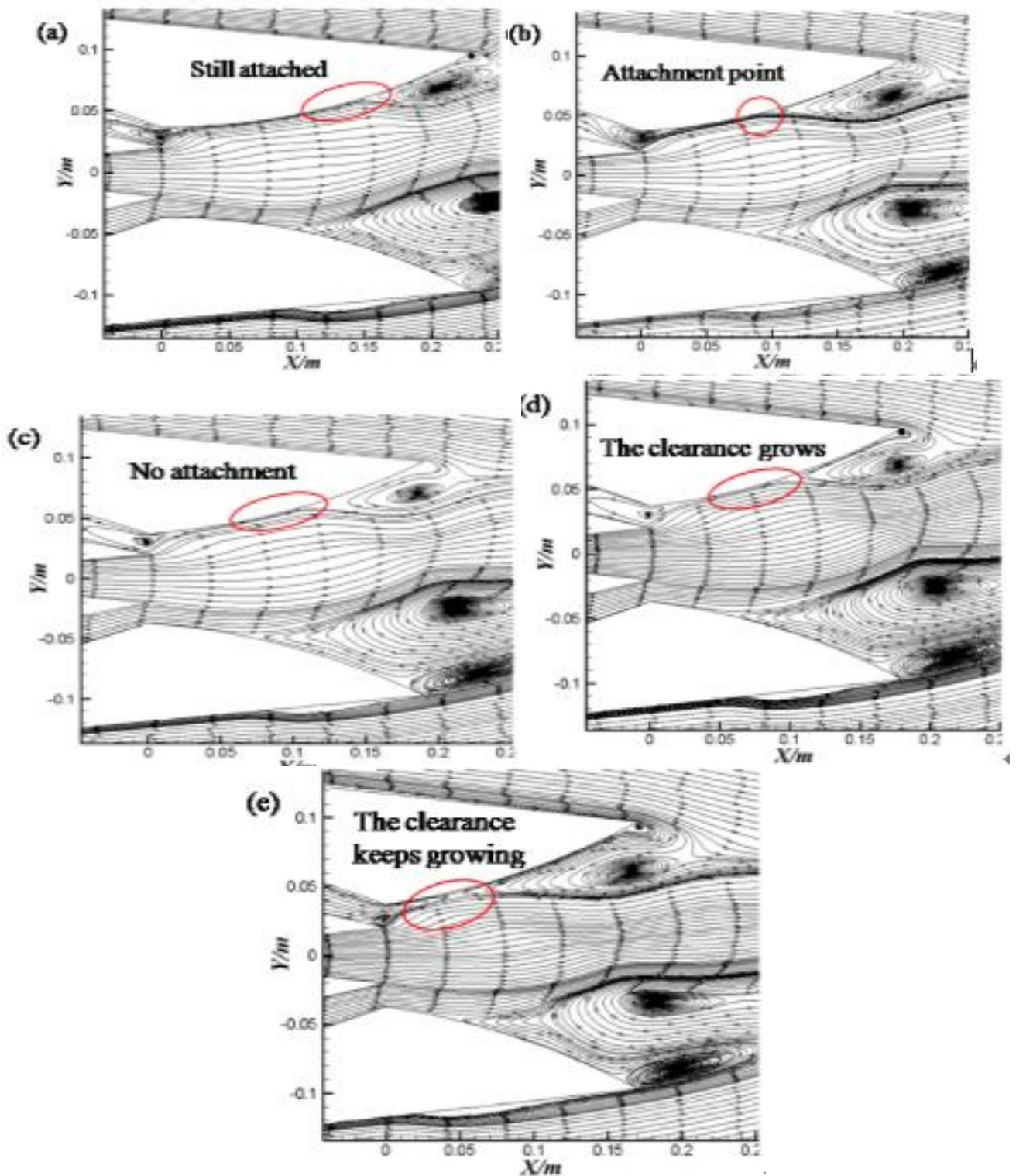

Figure 7. The streamline diagrams for different axial length of outer collar L with (a) $0.23 \mathrm{~m}$, (b)

$$
0.2 \mathrm{~m}, \text { (c) } 0.19 \mathrm{~m}, \text { (d) } 0.18 \mathrm{~m}, \text { (e) } 0.17 \mathrm{~m} \text {. }
$$

As shown in Figure 7, the main flow is still attached when L increases to $0.23 \mathrm{~m}$ while returns to normal when $\mathrm{L}$ decreases to $0.19 \mathrm{~m}$ or less. The clearance between the main flow and the upper collar wall turns larger as L decreases.

As the outlet width of outer collar remains unchanged, increasing L means the outer collar space becomes narrower. As a result, the main flow gets more limitations laterally, which is the Y-direction, and a lower angle for the main flow to be deflected with the influence of the pressure difference. So the main flow tends to be attached to the upper collar wall with a larger L under the same flow condition. On the contrary, the flow space becomes larger and the entrained outflow increases, which leads to a larger clearance and attachment is avoided. A critical axial length $\mathrm{L}$ is present to determine jet attachment and the critical value is between $0.19 \mathrm{~m}$ and $0.2 \mathrm{~m}$ for the model. Jet attachment can be avoided by decreasing the axial length of outer collar and the clearance increases as well. 


\section{Conclusions}

Numerical simulation of jet attachment of counter-flow thrust vectoring nozzle at zero attack and subsonic conditions is conducted and the effectiveness of the evasion method by varying the geometric parameters is verified. It can be concluded as follows.

1) When the suction pressure varies between 0.1 bar and $0.2 \mathrm{bar}$, the main flow is attached to the upper collar wall and the attachment point moves towards collar inlet as the suction pressure increases.

2) A critical slot width, between $0.017 \mathrm{~m}$ and $0.02 \mathrm{~m}$, is present and jet attachment can be avoided by increasing the slot width $\mathrm{G}$ and the clearance increases as well.

3) A critical axial length of outer collar, between $0.19 \mathrm{~m}$ and $0.2 \mathrm{~m}$, is also present and decreasing $\mathrm{L}$ is an effective evasion method to jet attachment.

\section{References}

1. Z.M. Liu, Y.L. Xu, F. Shen, J. Prop. Tech. 35, 3 (2014)

2. D. Dores, S.M. Madruga, A Krothapalli, et al, AIAA, 3516 (2006)

3. A. Jimenez, AIAA, 3991 (2001)

4. K.A. Deere, B.L. Berrier, J.D. Flamm, AIAA, 3502 (2005)

5. C.A. Hunter, K.A. Deere, AIAA, 2669 (1999)

6. J.D. Flamm, AIAA, 3255 (1998)

7. M.S. Wang, J.J. Yang, J. Aero. Power, 24, 1 (2009)

8. M.S. Wang, J.J. Yang, Acta Aero. Et Astr. Sin. 30,4 (2009)

9. Z.M. Liu, C. Chen, F. Shen, Y.L. Xu, J. Prop. Tech. 35,4 (2014)

10. Z.M. Liu, Y.L. Xu, F. Shen, J. Aero. Power, 29,6 (2014) 